Pacific Journal of Mathematics

A GENERAL VERSION OF VAN DER CORPUT'S DIFFERENCE 


\title{
A GENERAL VERSION OF VAN DER CORPUT'S DIFFERENCE THEOREM
}

\author{
RUdOLF J. TASCHNER
}

\begin{abstract}
Let $\omega(n)$ be a real-valued sequence, and let us assume that for all positive integers $g$ the difference-sequences $\Delta_{g} \omega(n)=\omega(n+g)-\omega(n)$ are uniformly distributed modulo 1 , then $\omega(n)$ itself is uniformly distributed modulo 1 . This is van der Corput's difference theorem or the so-called main theorem in the theory of uniform distribution. In this paper I present a rather generalized version of this theorem which not only enables us to prove the original van der Corput theorem, but also the general approximation theorem of Kronecker in its discrete and in its continuous version. Moreover, a few other examples of uniformly distributed sequences can be constructed by this general difference theorem.
\end{abstract}

The proof of the difference theorem was given by van der Corput in using his so-called "fundamental inequality". M. Tsuji, E. Hlawka, J. H. B. Kemperman and R. J. Taschner formulated stronger versions of this fundamental inequality and by doing this reached to new aspects of the difference theorem. A different approach to the difference theorem was investigated by J. Bass and J.-P. Bertrandias: they related it to the Bochner-Herglotz representation theorem about positive definite functions. This method was further elaborated by J. Cigler. In particular, Cigler proved the uniform distribution of $n \alpha$ modulo 1 for irrational $\alpha$ only by using his version of the difference theorem. Cigler tried to extend his theorem for uniformly distributed functions, defined on a local compact commutative group. He recognized, however, that the topology of the group troubles the application of the difference theorem. It is, for example, impossible to derive Hlawka's difference theorem about $C$-uniformly distributed functions by Cigler's method.

In this paper we will follow the way, indicated by Bass, Bertrandias, and Cigler, but we separate the domain of the uniformly distributed functions from the group which makes it possible to define difference sequences. By doing this, the difficulties raised by the topology of the group can be avoided.

Before formulating the main theorem, we list all necessary prerequisites: Let $X$ be a compact group and let $\chi$ be the normed Haar measure on $X . E^{h}, h \in H$, designates a "main system" of representations of $X$, this means: a set of irreducible unitary representations so that each irreducible 
unitary representation of $X$ is equivalent to one and only one member of the $E^{h}$. By $E^{0}$, i.e. if $h=0$, we designate the trivial representation: $E^{0}=1$.

Let $\Sigma$ be a measure space and $\sigma_{R}, R \in \Re$, a net of measures on $\Sigma$; $\Re$ designates an inductively ordered set. We postulate the following two assumptions: the $\sigma_{R}$ shall be uniformly bounded, i.e.

$$
\sup _{R \in \mathscr{R}} \sigma_{R}(\Sigma)<\infty
$$

and finally normed, i.e.

$$
\lim _{R \in \Re} \sigma_{R}(\Sigma)=1
$$

A measurable function $\omega: \Sigma \rightarrow X$ is called uniformly distributed, if for all $h \in H, h \neq 0$,

$$
\lim _{R \in \Re} \int_{\Sigma} E^{h}(\omega(s)) d \sigma_{R}(s)=0 .
$$

From this formula, it is easy to derive

$$
\lim _{R \in R} \int_{\Sigma} f(\omega(s)) d \sigma_{R}(s)=\int_{X} f(x) d \chi(x)
$$

for all continuous $f: X \rightarrow \mathbf{C}$, and the converse is true too.

Let $\Gamma$ be a local compact commutative group and let each $g \in \Gamma$ be a measurable function from $\Sigma$ into $\Sigma$, i.e. $g: \Sigma \rightarrow \Sigma$ so that for all $s \in \Sigma$ $g s \in \Sigma$ is well defined. $1 \in \Gamma$ stands for the unit. We postulate the following conditions: for all $g^{\prime}, g^{\prime \prime} \in \Gamma$ and all $s \in \Sigma$ we have $g^{\prime}\left(g^{\prime \prime} s\right)=$ $\left(g^{\prime} g^{\prime \prime}\right) s$, and $g^{\prime} s=g^{\prime \prime} s$ implies $g^{\prime}=g^{\prime \prime}$. If the limit

$$
\lim _{R \in \bar{\Re}} \int_{\Sigma} f(s) d \sigma_{R}(s)
$$

exists for a measurable and bounded $f: \Sigma \rightarrow \mathbf{C}$ and a cofinal $\overline{\mathcal{R}} \subseteq \Re$, then the limit

$$
\lim _{R \in \overline{\mathcal{R}}} \int_{\Sigma} f(g s) d \sigma_{R}(s)
$$

shall also exist for each $g \in \Gamma$ and coincide with the former limit. We assume the existence of a sequence $\gamma_{n}$ of measures on $\Gamma$, which is finally normed, i.e.

$$
\lim _{n \rightarrow \infty} \gamma_{n}(\Gamma)=1
$$


and finally translationinvariant, i.e. if the limit

$$
\lim _{n \rightarrow \infty} \int_{\Gamma} f(g) d \gamma_{n}(g)
$$

exists for a measurable and bounded $f: \Gamma \rightarrow \mathbf{C}$, then the limit

$$
\lim _{n \rightarrow \infty} \int_{\Gamma} f\left(g^{\prime} g\right) d \gamma_{n}(g)
$$

exists also for each $g^{\prime} \in \Gamma$ and coincides with the former one.

$\bar{\Gamma}$ designates the group of the characters $\bar{g}$ of $\Gamma ; \overline{1}$ stands for the trivial character. Instead of $\bar{g}(g)$ we write $(g, \bar{g})$.

For all functions $\omega: \Sigma \rightarrow X$ we tacitly assume that the functions $g \mapsto E^{h}(\omega(g s))$ are uniformly continuous in $s \in \Sigma$.

We can now formulate the main result:

Let $\omega: \Sigma \rightarrow X$ be a measurable function. We consider for each $g \in \Gamma$ the difference function $\Delta_{g} \omega(s)=\omega(s)^{-1} \omega(g s)$, and assume that

$$
\lim _{R \in \mathscr{R}} \int_{\Sigma} E^{h}\left(\Delta_{g} \omega(s)\right) d \sigma_{R}(s)
$$

exists for all $h \in H$. We construct a measure $\bar{\eta}^{h}$ on $\bar{\Gamma}$ with the property

$$
\lim _{R \in \Re} \int_{\Sigma} E^{h}\left(\Delta_{g} \omega(s)\right) d \sigma_{R}(s)=\int_{\bar{\Gamma}}(g, \bar{g}) d_{\bar{\eta}}^{h}(\bar{g}) .
$$

If $\bar{\eta}^{h}$ vanishes at the set consisting only of $\overline{1}$ for all $h \neq 0$, i.e. if $\bar{\eta}^{h}(\overline{1})=0$, then $\omega$ is uniformly distributed.

Before proving this theorem, we want to illustrate its value in several examples of applications:

(1) $X=\mathbf{R} / \mathbf{Z}, \chi=$ Lebesgue measure, $E^{h}(x)=e^{2 \pi i h x}, h \in \mathbf{Z} . \Sigma=\mathbf{Z}$. We define $\sigma_{R}$ to be the counting measure on the points $\{1, \ldots, R\}$, normed by the factor $R^{-1} . \Gamma=\mathbf{Z}$ with the addition as operation. If the sequence $\Delta_{g} \omega(n)=\omega(g+n)-\omega(n)$ is uniformly distributed for each $g \neq 0$, then we obtain for all $h \neq 0$

$$
\begin{aligned}
\int_{\bar{\Gamma}}(g, \bar{g}) d_{\bar{\eta}}^{h}(\bar{g}) & =\int_{0}^{1} e^{2 \pi i g \bar{g}} d_{\bar{\eta}}^{h}(\bar{g})=\lim _{R \rightarrow \infty} \frac{1}{R} \sum_{n=1}^{R} e^{2 \pi i h \Delta_{g} \omega(n)} \\
& = \begin{cases}1, & \text { if } g=0 \\
0, & \text { otherwise. }\end{cases}
\end{aligned}
$$


Thus $\bar{\eta}^{h}$ coincides with the Lebesgue measure and vanishes at the onepoint-set consisting of $0, \bar{\eta}^{h}(0)=0$. This proves van der Corput's difference theorem.

(2) All prerequisites as in (1), only $\Gamma=m \mathbf{Z}$ with an arbitrary positive integer $m$. The same inference as before establishes an assumption of $\mathrm{H}$. Delange: It is sufficient for the uniform distribution of $\omega(n)$ that the difference sequences $\Delta_{g} \omega(n)=\omega(g+n)-\omega(n)$ are uniformly distributed for all multiples $g=m n$ of a positive integer $m$.

(3) $X, \chi, E^{h}$ as in (1). $\Sigma=\mathbf{R}$. By defining $\sigma_{R}$ as Lebesgue measure on the interval $[0, R], R \in \mathbf{R}^{+}=\Re$, normed by the factor $R^{-1}$, we are dealing with the $C$-uniform distribution. We take $\Gamma=\mu \mathbf{Z}$ with an arbitrary $\mu \in \mathbf{R}^{+}$and the addition as operation. The same calculation as in (1) leads to Hlawka's difference theorem: If the difference functions $\Delta_{g} \omega(t)=$ $\omega(g+t)-\omega(t)$ are C-uniformly distributed for all numbers $g=\mu n, n \in \mathbf{N}$, then $\omega(t)$ itself is $C$-uniformly distributed.

(4) $X, \chi, E^{h}$ as in (1). $\Sigma=\Gamma$ designates a local compact but non-compact commutative group, $\sigma_{R}, R \in \mathbf{N}$, is a sequence of measures on $\Gamma$, which converges weakly to a normed translationinvariant measure on $\Gamma$. These are Cigler's requirements for his difference theorem about uniformly distributed functions. Thus it is a special case of our theorem.

(5) $X=\mathbf{R}^{L} / \mathbf{Z}^{L}, \chi=$ Lebesgue measure, $E^{h_{l}}\left(x_{l}\right)=e^{2 \pi i h_{l} x_{l}}, h_{l} \in \mathbf{Z}^{L} . l$ ranges in $\{1, \ldots, L\}$, and we are using Einstein's sum convention in terms like $h_{l} x_{l}$. Given a monotone non-decreasing sequence of natural numbers $S_{R}, R \in \mathbf{N}$, we define $\Sigma_{R}$ to be the set of all functions $\xi: \mathbf{N} \rightarrow \mathbf{N}_{0}$ with $\xi(n) \leq R$ for all $n \in \mathbf{N}$ and $\xi(n)=0$ for all $n>S_{R}$. We distinguish two types of examples: type $I$ is characterized by $\sup _{R} S_{R}=S<\infty$, type $I I$ by $\sup _{R} S_{R}=S=\infty$. Let $\Sigma$ be the set of all functions $\xi: \mathbf{N} \rightarrow \mathbf{Z}$ with $\xi(n)=0$ for all $n>S$ in the case of type I or with $\xi(n)=0$ for all $n$ greater than a certain index $n_{0}=n_{0}(\xi)$ in the case of type II. In any case, let $\sigma_{R}$ be the counting measure concentrated on $\Sigma_{R}$, and normed by the factor $(R+1)^{-S_{R}}$. $\Gamma=\mathbf{Z}$ with the addition as operation. For any $g \in \Gamma$ and $\xi \in \Sigma$, we define $g+\xi=\zeta$ by $\zeta(n)=\xi(n)$ for all $n$ except $n=q$, and $\zeta(q)=g+\xi(q)$. The choice of $q \in \mathbf{N}$ will be made afterwards, the only condition, $q$ must fulfill till now is $q \leq S$ in the case of type I. Let $\lambda_{l}(n), n=1,2, \ldots$ be a given sequence of points in $\mathbf{R}^{L}$ and consider the function

$$
\omega(\xi)=\sum_{n=1}^{\infty} \xi(n) \lambda_{l}(n) .
$$


The difference function $\Delta_{g} \omega(\xi)=\omega(g+\xi)-\omega(\xi)=g \lambda_{l}(q)$ is constant and by the formula

$$
\lim _{R \rightarrow \infty} \int_{\Sigma} e^{2 \pi i h_{l} \Delta_{g} \omega(\xi)} d \sigma_{R}(\xi)=e^{2 \pi i h_{l} \lambda_{l}(q) g}=\int_{0}^{1} e^{2 \pi i g \bar{g}} d_{\bar{\eta}}^{h_{l}}(\bar{g})
$$

we derive that $\bar{\eta}^{h_{l}}$ is a point measure, concentrated on $h_{l} \lambda_{l}(q)$ modulo 1 . The proof of the theorem will show that the choice of $\Gamma$ may depend on $h_{l}$; we only have to look for a $q \leq S$ with $h_{l} \lambda_{l}(q) \neq 0(\bmod 1) . \omega(\xi)$ is uniformly distributed modulo 1 , if for each $h_{l} \in \mathbf{Z}^{L}-\{0\}$ there exists a $q \in \mathbf{N}, q \leq S$, such that $h_{l} \lambda_{l}(q) \notin \mathbf{Z}$.

This is, in the case of type I, a proof of Kronecker's general approximation theorem in discrete variables. If $S=1$, we reach $\mathrm{H}$. Weyl's theorem about the uniform distribution of $n \lambda_{l}$ modulo 1 for linear independent $\lambda_{l}$ over $\mathbf{Z}$.

In the case of type II, all $\lambda_{l}(n)$ can be rational.

$$
\omega(\xi)=\sum_{n=1}^{\infty} \frac{\xi(n)}{n}
$$

is an example of an uniformly distributed function of this type.

(6) All prerequisites as in (5). We assume at least $S \geq 2$ and consider the function

$$
\omega(\xi)=\sum_{n=1}^{\infty} \xi(n) \xi(n+1) \lambda_{l}(n) .
$$

If we define $\xi(0)=0$, we have.

$$
\Delta_{g} \omega(\xi)=g\left(\xi(q-1) \lambda_{l}(q-1)+\xi(q+1) \lambda_{l}(q)\right)
$$

which is a function of the kind we discussed in (5). The condition $h_{l} \lambda_{l}(q) \notin \mathbf{Z}$ leads to

$$
\lim _{R \rightarrow \infty} \int_{\Sigma} e^{2 \pi i h_{l} \Delta_{g} \omega(\xi)} d \sigma_{R}(\xi)=\int_{0}^{1} e^{2 \pi i g \bar{g}} d_{\bar{\eta}^{\prime}}^{h_{l}}(\bar{g})= \begin{cases}1, & \text { if } g=0 \\ 0, & \text { otherwise }\end{cases}
$$

i.e. $\bar{\eta}^{h_{l}}$ is the Lebesgue measure and therefore vanishes at the one-point-set consisting of $0 . \omega(\xi)$ is uniformly distributed modulo 1 , if for each $h_{l} \in$ $\mathbf{Z}^{L}-\{0\}$ there exists a $q \in \mathbf{N}, q \leq S$, such that $h_{l} \lambda_{l}(q) \notin \mathbf{Z}$.

(7) The last two examples can be extended to the following case which we describe by type III: Let $\Sigma_{R}$ be the set of all functions $\xi: \mathbf{N} \rightarrow \mathbf{N}_{0}$ with $\xi(n) \leq R$ for all $n \in \mathbf{N}$. For any $r \in\{0,1, \ldots, R\}$ and any $n \in \mathbf{N}$ we 
define $E_{R}(n ; r)$ as the set of all $\xi \in \Sigma_{R}$ with $\xi(n)=r$. The sigmaalgebra on $\Sigma_{R}$ shall be generated by the finite intersections of the $E_{R}(n ; r)$ and the measure $\sigma_{R}$ is defined on $\Sigma_{R}$ in the following way: for any $r_{1}, \ldots, r_{K} \in$ $\{0,1, \ldots, R\}$ and $n_{1}, \ldots, n_{K}$ with $n_{k} \neq n_{m}$ for $k \neq m$, we have

$$
\sigma_{R}\left(E_{R}\left(n_{1} ; r_{1}\right) \cap \cdots \cap E_{R}\left(n_{K} ; r_{K}\right)\right)=(R+1)^{-K} \text {. }
$$

Let $\Sigma$ be the set of all bounded functions $\xi: \mathbf{N} \rightarrow \mathbf{Z}$. Analogue results to (5) and (6) can easily be derived, one has only to guarantee that

$$
\sum_{n=1}^{\infty} \xi(n) \lambda_{l}(n) \quad \text { resp. } \sum_{n=1}^{\infty} \xi(n) \xi(n+1) \lambda_{l}(n)
$$

converges (e.g. by postulating $\left|\lambda_{l}(n)\right| \leq \alpha^{n}$ with $0<\alpha<1$ ).

(8) $X, \chi, E^{h_{l}}\left(x_{l}\right)$ as in (5). Let $\Sigma_{R}$ be the set of all functions $\xi$ : $\mathbf{N} \rightarrow \mathbf{R}_{0}^{+}$with $\xi(n) \leq R$ for all $n \in \mathbf{N}$ and $\xi(n)=0$ for all $n>S_{R}$. Here $R$ ranges over $\Re=\mathbf{R}^{+}$, but as before $S_{R}$ designates a non-decreasing net of natural numbers. Let $\sigma_{R}$ be the Lebesgue measure concentrated on $[0, R]^{S_{R}}$ and normed by the factor $R^{-S_{R}}$. We define $\Sigma$ as the set of all functions $\xi$ : $\mathbf{N} \rightarrow \mathbf{R}$ with $\xi(n)=0$ for all $n>S$ in the case of type I resp. with $\xi(n)=0$ for all $n$ greater than a certain $n_{0}=n_{0}(\xi)$ in the case of type II. $\Gamma=\mu \mathbf{Z}$ with the addition as operation. $\mu$ is a positive real number which will be specified later. For any $g \in \Gamma$ and $\xi \in \Sigma$ we define $g+\xi=\zeta$ by $\zeta(n)=\xi(n)$ for all $n$ except $n=q$, and $\zeta(q)=g+\xi(q)$. The choice of $q \in \mathbf{N}, q \leq S$, will be made afterwards. Given a sequence of points $\lambda_{l}(n) \in \mathbf{R}^{L}$, we consider

$$
\omega(\xi)=\sum_{n=1}^{\infty} \xi(n) \lambda_{l}(n)
$$

The same calculation as in (5) shows that $\bar{\eta}^{h_{l}}$ is a point measure concentrated on $\mu h_{l} \lambda_{l}(q)$ modulo 1 . As $\mu$ is at our disposal, we only have to postulate $h_{l} \lambda_{l}(q) \neq 0$ for establishing $\mu_{l} \lambda_{l}(q) \notin \mathbf{Z}$. Thus we gain the following result: $\omega(\xi)$ is uniformly distributed modulo 1 , if for each $h_{l} \in$ $\mathbf{Z}^{L}-\{0\}$ there exists a $q \in \mathbf{N}, q \leq S$, such that $h_{l} \lambda_{l}(q) \neq 0$.

This is, in the case of type I, a proof of Kronecker's general approximation theorem in continuous variables. For $S=1$ we have as a special case the $C$-uniform distribution of $t \lambda_{l}$ for linear independent $\lambda_{l}$ over $\mathbf{Q}$.

(9) All prerequisites as in (8). We assume at least $S \geq 2$ and consider the function

$$
\omega(\xi)=\sum_{n=1}^{\infty} \xi(n) \xi(n+1) \lambda_{l}(n) .
$$


The same calculation as in (6) shows: $\omega(\xi)$ is uniformly distributed modulo 1 , if for each $h_{l} \in \mathbf{Z}^{L}-\{0\}$ there exists a $q \in \mathbf{N}, q \leq S$, such that $h_{l} \lambda_{l}(q) \neq 0$.

(10) $X=\mathbf{R} / \mathbf{Z}, \chi=$ Lebesque measure, $E^{h}(x)=e^{2 \pi i h x}, h \in \mathbf{Z} . \Sigma=$ $\mathbf{Q}^{+}, \Re=\mathbf{N}$, but ordered by the divisor relation. For each $R \in \mathbf{N}$ let $\sigma_{R}$ be the point measure concentrated on the positive divisors of $R$ and normed by dividing it by $\tau(R)$, the number of the positive divisors of $R$. Let $\Gamma$ be the set of numbers $g=p^{k}, k \in \mathbf{Z}, p$ a prime number, with the multiplication as operation. We consider the example

$$
\omega(n)=\log n .
$$

As $\Delta_{g} \omega(n)=\omega(g n)-\omega(n)=\log g=k \log p$, and as

$$
\lim _{R \in \mathcal{R}} \frac{1}{\tau(R)} \sum_{r \mid R} e^{2 \pi i h \Delta_{g} \omega(r)}=e^{2 \pi i h k \log p}=\int_{0}^{1} e^{2 \pi i k \bar{g}} d \bar{\eta}^{h}(\bar{g}),
$$

we see that $\bar{\eta}^{h}$ is concentrated at the point $h \log p$, which is $Z 0(\bmod 1)$ for all $h \in \mathbf{Z}, h \neq 0$. Therefore $\omega(n)=\log n$ is uniformly distributed in this sense of uniform distribution. It is well known that $\log n$ is not uniformly distributed in the usual sense of uniformly distributed sequences.

Now we come to the proof of the main theorem: Suppose $A: \Sigma \rightarrow \mathrm{C}^{N \cdot N}$ is a measurable and bounded function with range in the set of all $N \times N$-matrices. We derive the norm in $\mathbf{C}^{N \cdot N}$ from the inner product $(A \mid B)=\operatorname{trace}\left(B^{*} A\right)$. A function $\alpha: \Gamma \rightarrow \mathrm{C}$ is called correlation function of $A$, if there exists a cofinal $\bar{\Re} \subseteq \Re$ with

$$
\lim _{R \in \bar{\Re}} \int_{\Sigma}(A(g s) \mid A(s)) d \sigma_{R}(s)=\alpha(g)
$$

for all $g \in \Gamma$. The formulas $\alpha(1) \geq 0$ and $\alpha\left(g^{-1}\right)=\overline{\alpha(g)}$ are obvious. As $\rho=\sup _{s \in \Sigma}\|A(s)\|^{2} \cdot \sup _{R \in \Re} \sigma_{R}(\Sigma)<\infty$ and as $\{z \in \mathbf{C}:|z| \leq \rho\}^{\Gamma}$ is compact by Tychonoff's theorem,

$$
\left(\int_{\Sigma}(A(g s) \mid A(s)) d \sigma_{R}(s)\right)_{g \in \Gamma}, \quad R \in \Re,
$$

represents a net in a compact space. Thus there exists at least one correlation function of $A$. The set of all these correlation functions is designated by $\mathcal{C}(A)$. If $g \mapsto A(g s)$ is uniformly continuous in $s$, all $\alpha \in \mathcal{C}(A)$ are continuous. It is easy to check that all $\alpha \in \mathcal{C}(A)$ are positive definite. "Positive definite" means that a function $\varphi: \Gamma \rightarrow \mathbf{C}$ fulfills for any $g_{1}, \ldots, g_{N} \in \Gamma$ and $c_{1}, \ldots, c_{N} \in \mathbf{C}$ the condition

$$
\sum_{m, n=1}^{N} c_{m} \overline{c_{n}} \varphi\left(g_{m} g_{n}^{-1}\right) \geq 0 \text {. }
$$


Referring to a theorem of Raikov (cf. the book of M. A. Neumark), we can construct a measure $\bar{\alpha}$ on the character group $\bar{\Gamma}$ for each correlation function $\alpha$ so that

$$
\alpha(g)=\int_{\bar{\Gamma}}(g, \bar{g}) d \bar{\alpha}(\bar{g}) .
$$

In the case of discrete groups $\Gamma$-which we used in almost all of our examples-one only needs the theorem of Bochner and Herglotz for the same result. By using the formula

$$
\lim _{n \rightarrow \infty} \int_{\Gamma}(g, \bar{g}) d \gamma_{n}(g)= \begin{cases}1, & \text { if } \bar{g}=\overline{1} \\ 0, & \text { otherwise }\end{cases}
$$

we establish

$$
\lim _{n \rightarrow \infty} \int_{\Gamma} \alpha(g) d \gamma_{n}(g)=\bar{\alpha}(\overline{1}) \geq 0 .
$$

The exchange of integral and limit is justified by Lebesgue's theorem about dominated convergence. If we have for some cofinal $\bar{R} \subseteq \Re$

$$
\lim _{R \in \overline{\mathcal{R}}} \int_{\Sigma} A(s) d \sigma_{R}(s)=M,
$$

we can derive

$$
\|M\|^{2} \leq \varlimsup_{\alpha \in \mathcal{C}_{(A)}} \bar{\alpha}(\overline{1}) .
$$

We prove this by defining $B=A-M$, considering the formulas

$$
\lim _{R \in \bar{\Upsilon}} \int_{\Sigma} B(s) d \sigma_{R}(s)=0,
$$

$$
(A(g s) \mid A(s))=\|M\|^{2}+(M \mid B(s))+(B(g s) \mid M)+(B(g s) \mid B(s)),
$$

and concluding $\alpha(g)=\|M\|^{2}+\beta(g)$ for any $\alpha \in \mathcal{C}(A)$ with a suitable $\beta \in \mathcal{C}(B)$ so that

$$
\begin{aligned}
\varlimsup_{\alpha \in \mathcal{C}(A)} \bar{\alpha}(\overline{1}) & \geq \lim _{n \rightarrow \infty} \int_{\Gamma} \alpha(g) d \gamma_{n}(g)=\|M\|^{2}+\lim _{n \rightarrow \infty} \int_{\Gamma} \beta(g) d \gamma_{n}(g) \\
& =\|M\|^{2}+\bar{\beta}(\overline{1}) \geq\|M\|^{2},
\end{aligned}
$$

which establishes the asserted formula. If $\overline{\mathcal{C}}(A)$ represents the set of all measures $\bar{\alpha}$, corresponding to $\alpha \in \mathcal{C}(A)$, and if $\bar{\alpha}(\overline{1})=0$ for all $\bar{\alpha} \in \bar{C}(A)$, then we have

$$
\lim _{R \in \Re} \int_{\Sigma} A(s) d \sigma_{R}(s)=0
$$


Now we specialize to $A(s)=E^{h}(\omega(s))$. Let $\eta^{h}$ be the elements of $\mathcal{C}\left(E^{h}(\omega)\right)$ and let $\bar{\eta}^{h}$ be the measures of $\bar{C}\left(E^{h}(\omega)\right)$. From the former arguments we imply (by simply writing $E^{h}\left(\Delta_{g} \omega\right)$ instead of $\left.\left(E^{h}\left(\Delta_{g} \omega\right) \mid E^{0}\right)\right)$

$$
\lim _{R \in \Re} \int_{\Sigma} E^{h}\left(\Delta_{g} \omega(s)\right) d \sigma_{R}(s)=\int_{\bar{\Gamma}}(g, \bar{g}) d \bar{\eta}^{h}(\bar{g}),
$$

if the limit on the left side exists, and

$$
\lim _{R \in \mathscr{R}} \int_{\Sigma} E^{h}(\omega(s)) d \sigma_{R}(s)=0,
$$

if $\bar{\eta}^{h}(\overline{1})=0$. This proves the theorem.

\section{REFERENCES}

1. J. Bass, Suites uniformément denses, moyennes trigonométriques, fonctions pseudo-aléatoires, Bull. Soc. Math. France, 87 (1959), 1-64.

2. J. Bass and J.-P. Bertrandias, Moyennes de sommes trigonométriques et fonctions d'autocorrélation, C.R. Acad. Sci. Paris, 245 (1957), 2457-2459.

3. J. Cigler, Ueber eine Verallgemeinerung des Hauptsatzes der Theorie der Gleichverteilung, J. reine angew. Math., 210 (1962), 141-147.

4. J. G. van der Corput, Diophantische Ungleichungen I. Zur Gleichverteilung modulo Eins, Acta Math., 56 (1931), 373-456.

5. E. Hlawka, Zur formalen Theorie der Gleichverteilung in kompakten Gruppen, Rend. Circ. Mat. Palermo, 4 (1955), 33-47.

6. __ Zum Hauptsatz der Theorie der Gleichverteilung, Oesterr. Akad. Wiss., Anz. math. -nat. Kl. (1957), 313-317.

7. _ Ueber C-Gleichverteilung, Ann. Mat. Pura Appl., 49 (1960), 311-326.

8. _ Theorie der Gleichverteilung, Mannheim, 1979.

9. J. H. B. Kemperman, On the distribution of a sequence in a compact group, Compositio Math., 16 (1964), 138-157.

10. L. Kuipers and H. Niederreiter, Uniform Distribution of Sequences, New York, 1974.

11. M. A. Neumark, Normierte Algebren, Berlin, 1959.

12. R. J. Taschner, Der Differenzensatz von van der Corput und gleichverteilte Funktionen, Oesterr. Akad. Wiss., Anz. math. -naturw. Kl. (1978), 115-118.

13. ,Der Differenzensatz von van der Corput und gleichverteilte Funktionen, J. Reine Angew. Math.

14. M. Tsuji, On the uniform distribution of numbers mod 1, J. Math. Soc. Japan, 4 (1952), 313-322.

15. H. Weyl, Ueber die Gleichverteilung von Zahlen mod. Eins, Math. Ann., 77 (1916), $313-352$.

Received September 2, 1979. This paper was written while the author was a visiting scholar at Stanford University. He wants to express his gratitude to the Mathematics Department of Stanford University for its hospitality.

STANFORD UNIVERSITY

STANFORD, CA 94305

Current Address: Inst. f. Math. Analysis, TU Wien

Gusshausstr. 27-29, A-1040 Wien/Vienna

Oesterreich/Austria 



\section{PACIFIC JOURNAL OF MATHEMATICS \\ EDITORS}

DONALD BABBITT (Managing Editor)

University of California

Los Angeles, CA 90024

\section{Hugo Rossi}

University of Utah

Salt Lake City, UT 84112

C. C. Moore and Arthur Ogus

University of California

Berkeley, CA 94720
J. DugundiI

Department of Mathematics

University of Southern California

Los Angeles, CA 90089-1113

R. FINN and H. SAMELSON

Stanford University

Stanford, CA 94305

ASSOCIATE EDITORS
R. ARens
E. F. BECKENBACH
B. H. NeumanN
F. WOLF
K. YoshidA (1906-1982)

\section{SUPPORTING INSTITUTIONS}

UNIVERSITY OF ARIZONA

UNIVERSITY OF BRITISH COLUMBIA

CALIFORNIA INSTITUTE OF TECHNOLOGY

UNIVERSITY OF CALIFORNIA

MONTANA STATE UNIVERSITY

UNIVERSITY OF NEVADA, RENO

NEW MEXICO STATE UNIVERSITY

OREGON STATE UNIVERSITY
UNIVERSITY OF OREGON

UNIVERSITY OF SOUTHERN CALIFORNIA

STANFORD UNIVERSITY

UNIVERSITY OF HAWAII

UNIVERSITY OF TOKYO

UNIVERSITY OF UTAH

WASHINGTON STATE UNIVERSITY

UNIVERSITY OF WASHINGTON 


\section{Pacific Journal of Mathematics}

Vol. 104, No. 1

May, 1983

Nestor Edgardo Aguilera and Eleonor Ofelia Harboure de Aguilera, On

the search for weighted norm inequalities for the Fourier transform $\ldots \ldots .1$

Jin Akiyama, Frank Harary and Phillip Arthur Ostrand, A graph and its complement with specified properties. VI. Chromatic and achromatic numbers ......................................... 15

Bing Ren Li, The perturbation theory for linear operators of discrete type . . . 29

Peter Botta, Stephen J. Pierce and William E. Watkins, Linear

transformations that preserve the nilpotent matrices .............. 39

Frederick Ronald Cohen, Ralph Cohen, Nicholas J. Kuhn and Joseph

Alvin Neisendorfer, Bundles over configuration spaces .......... 47

Luther Bush Fuller, Trees and proto-metrizable spaces . . . . . . . . . . 55

Giovanni P. Galdi and Salvatore Rionero, On the best conditions on the

gradient of pressure for uniqueness of viscous flows in the whole space . . 77

John R. Graef, Limit circle type results for sublinear equations $\ldots \ldots \ldots \ldots 85$

Andrzej Granas, Ronald Bernard Guenther and John Walter Lee,

Topological transversality. II. Applications to the Neumann problem for

$y^{\prime \prime}=f\left(t, y, y^{\prime}\right) \ldots \ldots \ldots \ldots \ldots \ldots \ldots \ldots \ldots \ldots \ldots \ldots \ldots \ldots \ldots . \ldots 5$

Richard Howard Hudson and Kenneth S. Williams, Extensions of

theorems of Cunningham-Aigner and Hasse-Evans . . . . . . . . . . . 111

John Francis Kurtzke, Jr., Centralizers of irregular elements in reductive algebraic groups

James F. Lawrence, Lopsided sets and orthant-intersection by convex

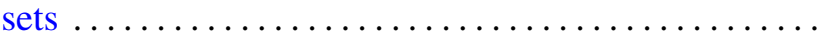

Åsvald Lima, G. H. Olsen and U. Uttersrud, Intersections of $M$-ideals and

$G$-spaces

Wallace Smith Martindale, III and C. Robert Miers, On the iterates of derivations of prime rings

Thomas H. Pate, Jr, A characterization of a Neuberger type iteration procedure that leads to solutions of classical boundary value problems

Carl L. Prather and Ken Shaw, Zeros of successive iterates of multiplier-sequence operators

Billy E. Rhoades, The fine spectra for weighted mean operators

Rudolf J. Taschner, A general version of van der Corput's difference theorem

Johannes A. Van Casteren, Operators similar to unitary or selfadjoint ones 